\title{
Axiomatic Characterization of Insurance Prices
}

\author{
Shaun S. Wanga $\quad$ Virginia R. Young ${ }^{b}$ Harry H. Panjer ${ }^{a}$ \\ ${ }^{a}$ Dept of Statiatics and Actuarial Science, University of Waterloo, ON, Canada N2L 3G1 \\ ${ }^{b}$ School of Business, University of Wisconsin-Madison, Madison, Wisconsin, USA 53706
}

\begin{abstract}
In this paper, we take an axiomatic approach to characterize insurance prices in a competitive market setting. We present four axioms to describe the behavjor of market insurance prices. From these axioms it follows that the price of an insurance risk has a Choquet integral representation with respect to a distorted probability, (Yaari, 1987). We propose an additional axiom for reducing compound risks. This axiom determines that the distortion function is a power function.
\end{abstract}

Keywords: Integral representation; Insurance pricing; Choquet integral.

\section{Introduction}

In this paper we take an axiomatic approach to characterize insurance prices. By insurance price, or insurance premium, we refer to the pure premium for an insurance risk which includes the net expected loss plus a risk load charge, excluding expenses and commissions. We shall concentrate on market prices, rather than premiums that would have been charged if an individual insurer were acting alone without competition. It is argued that in a competitive market, insurance prices are determined by the collective efforts of all agents (buyers and sellers). Under this argument, individual insurers are not price-makers, but price-takers (Meyers, 1991); i.e., the insurance price cannot be influenced by the actions of a single insurer.

An axiomatic approach has been at the heart of economic theories of risk and uncertainty. The five axioms of individual risk preferences in von Neumann and Morgenstern 
(1947) laid the foundation for expected utility (EU) theory. Yaari (1987) proposes a dual axiom to the independence axiom in EU and, thus, establishes a competing dual theory for risk and uncertainty. ${ }^{1}$ In Yaari's dual theory, the certainty equivalent of a uniformly bounded economic prospect $V,(0 \leq V \leq 1)$, can be represented as a Choquet integral:

$$
\int_{0}^{1} g\left[S_{V}(t)\right] d t
$$

where $S_{V}(t)=\operatorname{Pr}\{V>t\}, 0 \leq t \leq 1$, is the decumulative distribution function of $V$ and $g$ is an increasing function with $g(0)=0$ and $g(1)=1$.

In this paper, we first present four axioms to describe the behavior of insurance prices in a competitive market. As a refinement of a mathematical result of Greco (see Denneberg, 1994), we show that from these axioms it follows that the price for an insurance risk has a Choquet integral representation with respect to a distorted probability. This extends the result of Yaari (1987) to unbounded random variables. This paper also contributes by

- Providing market interpretations of insurance prices and axioms for insurance price behaviors. This differs from the economic theories of Yaari (1987) and Schmeidler (1989) which concern individual risk preferences.

- Furthering the axiomatic approach by adding an additional axiom for reducing compound risks. This axiom determines that the distortion function is a power function. This provides a theoretical justification for the PH-transform method in insurance pricing (Wang, 1995, 1996a,b).

- Describing how our results are analogous with the principle of no arbitrage.

\section{Market premium functional}

Let $\mathcal{X}$ be the set of non-negative random variables which represent the random losses associated with insurance contracts. We can think of a risk $X \in \mathcal{X}$ as a measurable nonnegative real valued function on an underlying measure space $(\Omega, \mathcal{A}, P)$, in which $\mathcal{A} \subset 2^{\Omega}$ is a $\sigma$-algebra and $P$ is a probability measure. The set $\Omega$ is the collection of outcomes, or

\footnotetext{
${ }^{1}$ For an account of the axioms in EU and Yaari's dual theory, see Quiggin (1993).
} 
states of the world, and the $\sigma$-algebra of subsets $\mathcal{A}$ is the collection of events. Thus, market premiums can be described as a functional from the set of insurance risks to the extended non-negative real numbers

$$
\mathrm{H}: \boldsymbol{X} \rightarrow[0, \infty]
$$

In this section we propose some axioms for this functional.

Axiom 1 (Conditional State Independence) For a given market condition, the price of an insurance risk $X$ depends only on its distribution.

We will use the decumulative distribution function (ddf) (or survival function) to characterize the distribution of $X$. The ddf of $\mathrm{X}$ is denoted as

$$
S_{X}(t)=P\{\omega: X(\omega)>t\}, \quad t \geq 0
$$

The price charged for a risk depends on the given market condition in some way. The market condition reflects the character of the market as a whole. For example, in a perfect market, with infinitely many players, perfect information and complete diversifiability of risks, the price should be the expected value, since the market should not provide rewards for diversifiable risks. When markets are incomplete, imperfect, and risks are not diversifiable without cost, the market will allow prices in excess of the expected value to reflect these conditions. This can lead to different prices in different markets with different degrees of incompleteness, imperfection and diversifiability. However, two risks with identical distributions in the same market should have identical prices because the probability distribution of the losses should determine the price, not the particular state of the world that leads to a given loss. Therefore, under Axiom 1, the market premium function $H$ satisfies the property that $\mathrm{H}[X]=\mathrm{H}\left[X^{\prime}\right]$ if $S_{X}=S_{X^{\prime}}$.

We now provide a basic axiom for comparing risks.

Axiom 2 (Monotonicity) For two risks $X$ and $Y$ in $\mathcal{X}$, if $X(\omega) \leq Y(\omega)$, for all $\omega \in \Omega$, a.s., then $\mathrm{H}[X] \leq \mathrm{H}[Y]$.

This axiom is reasonable because if a risk $Y$ results in a larger insurance claim than risk $X$ in (almost surely) every state of the world, then the insurance price for $Y$ should be greater than the price for $X$. 
Besides the concept of monotonicity, another useful concept for comparing risks is the stochastic dominance.

Definition 1 For two risks $X$ and $Y$ in $\mathcal{X}, X$ is said to precede $Y$ in first stochastic dominance (FSD) if $S_{X}(t) \leq S_{Y}(t)$ for all $t \geq 0$.

Kaas et al. (1994, Theorem 1.2, pp. 14-15) show the following relationship between monotonicity and FSD.

Lemma 1 If $X$ precedes $Y$ in $F S D$, then there exists a random variable $Y^{\prime \prime}$ with $S_{Y^{\prime}}=S_{Y}$ such that $X \leq Y^{\prime}$ with probability one.

As an immediate application of Lemma 1, we have the following result.

Proposition 1 If $\mathrm{H}$ satisfies Axioms 1 and 2, then $\mathrm{H}$ preserves FSD. In other words, for two risks $X$ and $Y$ in $\mathcal{X}$, if $S_{X}(t) \leq S_{Y}(t)$ for all $t \geq 0$, then $\mathrm{H}[X] \leq \mathrm{H}[Y]$.

Hadar and Russell (1969) give the following equivalent conditions for FSD.

1. $S_{X}(t) \leq S_{Y}(t)$, for all $t \geq 0$. That is, for every $t$, risk $Y$ has a higher tail probability.

2. For every decision-maker with an increasing utility function $u$ : $\mathrm{E}[u(-X)] \geq \mathrm{E}[u(-Y)]$. That is, every individual who thinks more wealth is better views $Y$ as more risky than $X$.

3. $Y$ is derived from $X$ by the addition of a random variable that is non-negative with probability one. That is, $Y$ equals $X$ plus an additional risk.

If one considers any of these three equivalent conditions, it is natural for the market to view $Y$ as being more risky than $X$. Hence, Axioms 1 and 2 require that the market premium for risk $Y$ will be greater than the one for risk $X$.

It is important to describe how the premium functional $\mathbf{H}$ operates when adding two risks. To do so, we use the concept of comonotonicity of risks. 
Definition 2 Let $X$ and $Y$ be random variables belonging to $\mathcal{X}$. We say that $X$ and $Y$ are comonotonic if, and only if, the inequality

$$
\left[X\left(\omega_{1}\right)-X\left(\omega_{2}\right)\right]\left[Y\left(\omega_{1}\right)-Y\left(\omega_{2}\right)\right] \geq 0
$$

holds almost surely (a.s.) for $\omega_{1}$ and $\omega_{2}$ in $\Omega$.

The term comonotonic comes from 'common monotonic' and is discussed by Schmeidler (1986) and Yaari (1987). The following equivalent condition is given by Denneberg (1994, pp. 54-55).

Proposition 2 Let $X$ and $Y$ belong to $\mathcal{X}$. Then $X$ and $Y$ are comonotonic if, and only if, there exists $Z \in \mathcal{X}$ and increasing real functions $f$ and $g$ such that

$$
X=f(Z), \quad Y=g(Z)
$$

Yaari (1987, pp.104) states the following about the concept of comonotonicity:

- "comonotonicity is a distribution-free property, in the sense that it is invariant under changes in the underlying measure. It is, in fact, an analogue of perfect correlation for this distribution-free setting. When two random variables are comonotonic, then it can be said that neither of them is hedge against the other. The variability of one is never tempered by counter-variability of the other."

If $X$ and $Y$ are comonotonic, the outcomes of $X$ and $Y$ always move in the same direction (good or bad), thus there is no hedge or diversifiability when pooling the two risks. For instance, quota shares or excess-of-loss layers of the same risk are comonotonic.

In a competitive market, Venter (1991) claims that additivity of insurance prices is required to avoid systematic arbitrage opportunities. However, strict additivity fails to explain the pooling effect, which is believed to be the essence of insurance (e.g. Albrecht, 1992). Therefore, instead of requiring additivity for all risks, we impose a weaker constraint by requiring additivity for comonotonic risks only. For comonotonic risks $X$ and $Y$, let $W=X+Y$. The market price for $W$ is at least the sum $\mathrm{H}[X]+\mathrm{H}[Y]$ because of the no-hedge condition (or non-diversifiability). On the other hand, the price for $W$ cannot 
exceed $\mathrm{H}[X]+\mathrm{H}[Y]$, because otherwise the policyholder can just buy separate insurance policies from different insurers.

Axiom 3 (Comonotonic Additive) If $X$ and $Y$ in $\mathcal{X}$ are comonotonic, then

$$
\mathrm{H}[\mathrm{X}+\mathrm{Y}]=\mathrm{H}[\mathrm{X}]+\mathrm{H}[\mathrm{Y}]
$$

We also propose that the market premium functional $\mathrm{H}$ satisfies two mild continuity properties:

Axiom 4 (Continuity) For $X \in \mathcal{X}$ and $d \geq 0$, the functional $\mathrm{H}$ satisfies

$$
\lim _{d \rightarrow 0^{+}} \mathrm{H}\left[(X-d)_{+}\right]=\mathrm{H}[X]
$$

and

$$
\lim _{d \rightarrow \infty} \mathrm{H}[\min (X, d)]=\mathrm{H}[X],
$$

in which $(X-d)_{+}=\max (X-d, 0)$.

In Axiom 4, the first condition says that a small truncation in the loss variable results in a small change in premium; the second condition says that $\mathrm{H}$ can be calculated by approximating $X$ by bounded variables. The continuity property has also been applied by Hürlimann (1994, Theorem 4.1).

\section{Choquet integral representation}

Consider a set function $\gamma: \mathcal{A} \rightarrow[0, \infty)$. Assume that the set function $\gamma$ is finite, zero on the empty set, and monotone; that is, $\gamma(\phi)=0, \gamma(\Omega)<\infty$, and

$$
A, B \in \mathcal{A}, \quad A \subset B \Longrightarrow \gamma(A) \leq \gamma(B)
$$

For a non-negative, real-valued random variable $X$, the Choquet integral of $X$ with respect to $\gamma$ can be evaluated as

$$
\int X d \gamma=\int_{0}^{\infty} \gamma\{\omega: X(\omega)>t\} d t
$$


Definition 3 Let $P$ be a probability measure on a $\sigma$-algebra $\mathcal{A}$ in $2^{n}$. For an increasing function $g$ on $[0,1]$ with $g(0)=0$ and $g(1)=1$, the set function $\gamma=g \circ P$ is called $a$ distorted probability and the function $g$ is called $a$ distortion function.

Under the distorted probability $\gamma=g \circ P$, the Choquet integral of $X \in \mathcal{X}$ can be evaluated as

$$
\int X d \gamma=\int_{0}^{\infty} g \circ P\{\omega: X(\omega)>t\} d t=\int_{0}^{\infty} g\left[S_{X}(t)\right] d t
$$

Theorem 1 (Greco) If the market premium functional

$$
\mathrm{H}: \boldsymbol{X} \rightarrow[0, \infty]
$$

satisfies Axioms 2-4, then there is a unique monotone set function $\gamma$ on $\mathcal{A}$, such that

$$
\mathbf{H}[X]=\int X d \gamma
$$

The set function $\gamma$ is given by $\gamma(A)=\mathrm{H}\left[I_{A}\right], A \in \mathcal{A}$.

Proof: This representation theorem was proved by Greco (Denneberg, 1994).

In fact, if we also assume that $H$ satisfies Axiom 1 , then we can write $\gamma$ as a distorted probability, $g \circ P$; however, $g$ will not necessarily be unique. If we impose a constraint on the collection of risks $\mathcal{X}$, then we can show that $g$ is unique, and we have the following theorem.

Theorem 2 Assume that the collection of risks $\mathcal{X}$ contains all the Bernoulli(u) random variables, $0 \leq u \leq 1$. If the market premium functional

$$
\mathrm{H}: \boldsymbol{X} \rightarrow[0, \infty]
$$

satisfies Axioms 1-4, then there is a unique distortion function $g$ such that

$$
\mathrm{H}[X]=\mathrm{H}[1] \int X d(g \circ P)=\mathrm{H}[1] \int_{0}^{\infty} g\left[S_{X}(t)\right] d t
$$

in which 1 represents the degenerate random variable which equals 1 with probability one. 
Proof: By Theorem 1, there exists a unique monotone set function $\gamma$ on $\mathcal{A}$, such that

$$
H[X]=\int X d \gamma
$$

The monotone set function $\gamma$ is given by $\gamma(A)=H\left[I_{A}\right], A \in \mathcal{A}$. We now only need to show that $\gamma=\mathrm{H}[1](g \circ P)$ in which $g$ is a unique distortion.

Step 1. Assume that $\mathrm{H}[\mathbf{1}]=1$.

Define $g:[0,1] \rightarrow[0,1]$ by $g(u)=\mathbf{H}\left[I_{u}\right]$ in which $I_{u}$ is a $\operatorname{Bernoulli}(u)$ random variable, $u \in[0,1]$, with

$$
P\left\{\omega: I_{u}(\omega)=0\right\}=1-u, \quad P\left\{\omega: I_{u}(\omega)=1\right\}=u
$$

Note that $\mathrm{H}\left[I_{u}\right]=\gamma\left\{\omega: I_{u}(\omega)=1\right\}$ by Theorem 1. Also note that, by Axiom $1, g$ is well-defined.

To show that $g$ is a distortion, first observe that $g(0)=\mathrm{H}\left[I_{0}\right]=0$. Indeed, because $I_{0}$ is comonotonic with respect to all random variables $X, \mathrm{H}[X]=\mathrm{H}\left[I_{0}+X\right]=\mathrm{H}\left[I_{0}\right]+\mathrm{H}[X]$ which implies that $\mathrm{H}\left[I_{0}\right]=0$. Second, $g$ is increasing because $H$ preserves FSD. Finally, $g(1)=\mathrm{H}\left[I_{1}\right]=1$, by assumption. Therefore, $g$ is a distortion on $[0,1]$.

We have defined $g$ so that for $0 \leq t<1$,

$$
\gamma\left\{\omega: I_{u}(\omega)>t\right\}=\gamma\left\{\omega: I_{u}(\omega)=1\right\}=g(u)=g\left(S_{I_{u}}(t)\right)
$$

and for $t \geq 1$,

$$
\gamma\left\{\omega: I_{u}(\omega)>t\right\}=\gamma(\phi)=0=g(0)=g\left(S_{I_{u}}(t)\right) .
$$

We want to show that $\gamma\{\omega: X(\omega)>t\}=g\left[S_{X}(t)\right]$ for all $X \in \mathcal{X}$ and $t \geq 0$.

For a given $X \in \mathcal{X}$ and for a fixed $t \geq 0, S_{X}(t) \in[0,1]$, so define a Bernoulli random variable $I_{S_{X}(z)}: \Omega \rightarrow[0, \infty]$ by

$$
I_{S_{X}(t)}(\omega)= \begin{cases}0, & \text { if } X(\omega) \leq t \\ 1, & \text { if } X(\omega)>t\end{cases}
$$

Then,

$$
\gamma\{\omega: X(\omega)>t\}=\gamma\left\{\omega: I_{S_{X}(t)}(\omega)=1\right\}=g\left[S_{X}(t)\right]
$$

and it follows that

$$
\mathrm{H}[X]=\int X d \gamma=\int_{0}^{\infty} \gamma[\omega: X(\omega)>t\} d t=\int_{0}^{\infty} g\left[S_{X}(t)\right] d t .
$$


Step 2. Drop the assumption of $H[1]=1$ and consider the premium functional $H^{\prime}[X]=H[X] / H[1]$. Note that if $H$ satisfies Axioms 1-4 then so does $H^{\prime}$.

Another plausible requirement on the premium functional is so-called no unjustified risk loading: For a degenerate variable $X=1$ with probability one, the insurance price is $\mathrm{H}[X]=1$.

Theorem 3 Assume that the collection of risks $\mathcal{X}$ contains all the Bernoulli(u) random variables, $0 \leq u \leq 1$. The premium functional $\mathrm{H}$ satisfies Axioms $1-4$ and $\mathrm{H}[X]=1$ if, and only if, $\mathbf{H}$ has a Choquet integral representation:

$$
\mathrm{H}[X]=\int X d(g \circ P)=\int_{0}^{\infty} g\left[S_{X}(t)\right] d t,
$$

where $g$ is increasing with $g(0)=0$ and $g(1)=1$. Furthermore, the following properties hold:

- Non-negative loading: $\mathrm{E}[X] \leq \mathrm{H}[X]$, for all $X$, if and only if $g(u) \geq u$, for all $u \in[0,1]$.

- Non-excessive loading: $\mathrm{H}[X] \leq \max (X)$.

- Scale and translation invariant: $\mathrm{H}[a X+b]=a \mathrm{H}[X]+b$, for $a \geq 0, b \geq 0$.

- Sub-additivity: If $g$ is concave, $\mathrm{H}[X+Y] \leq \mathrm{H}[X]+\mathrm{H}[Y]$.

- If $g$ is concave, then $\mathrm{H}$ preserves the second stochastic dominance. That is,

$$
\int_{x}^{\infty} S_{X}(t) d t \leq \int_{x}^{\infty} S_{Y}(t) d t, \text { for all } x \geq 0 \Longrightarrow \mathrm{H}[X] \leq \mathrm{H}[Y] .
$$

Proof: The "only if" part is a direct application of Theorem 2. For a proof of the "if" part as well as the listed properties see Denneberg (1994, pp. 64 and 71) and Wang (1996a).

Theorem 3 says that market prices are just expectations with respect to a new measure; however, this measure is not necessarily additive. This theorem is essentially Yaari's Representation Theorem (1987) extended to unbounded random variables. Also, we can extend Theorems 1-3 to represent a premium functional on the collection of real-valued random variables $\boldsymbol{X}$, Appendix A. For a real-valued loss variable, the negative part refers to a gain; for instance, in life insurance the loss variable can assume both positive and negative values for periodic premium payments. 


\section{Valuation of compound Bernoulli risks}

Consider a compound Bernoulli risk $X=I Y$, where $I$ is a Bernoulli frequency random variable with probability of occurrence $q=\operatorname{Pr}\{I=1\}=\operatorname{Pr}\{X>0\}$, and $Y$ represents the loss severity random variable given that a loss has occurred (that is, $Y=X \mid X>0$ ).

Suppose that two parties $A$ and $B$ have entered the following agreement: In the event that a loss occurs $(I=1)$, party A pays the market premium $\mathrm{H}[Y]$ to $\mathrm{B}$, and in exchange, party $B$ pays the actual loss amount $Y$. In this mutual agreement, party $A$ faces a contingent payout of $I \mathrm{H}[Y]$, and party $\mathrm{B}$ faces a contingent payout of $I Y$. In order to avoid any arbitrage opportunities, it is necessary that the market evaluates these two contingent payouts as having the same price. Thus, to evaluate the compound risk $I Y$, one can substitute the market price $\mathrm{H}[Y]$ for the loss severity variable $Y$. It follows that the compound risk $I Y$ is reduced to a one-stage risk, $Z=I \mathrm{H}[Y]$ with $\operatorname{Pr}\{Z=0\}=1-q$ and $\operatorname{Pr}\{Z=\mathrm{H}[Y]\}=q$.

In summary, we put forward the following axiom.

Axiom 5 (Reduction of Compound Bernoulli Risks) Let $X=I Y$ be a compound Bernoulli risk, where the Bernoulli frequency random variable $I$ is independent of the loss severity random variable $Y=X \mid X>0$. Then the market prices for risks $X=I Y$ and $I \mathrm{H}[Y]$ are equal.

Theorem 4 Assume that the collection of risks $\mathcal{X}$ contains all the Bernoulli random variables. Then the market premium functional $\mathrm{H}$ satisfies Axioms 1-5 if, and only if, $\mathrm{H}$ can be represented as

$$
\mathrm{H}[X]=\int_{0}^{\infty}\left[S_{X}(t)\right]^{r} d t
$$

where $r$ is some unique positive constant.

Proof: The "if" part. Note that for $X=I Y$ and $I \sim \operatorname{Bernoulli}(u), 0 \leq u \leq 1$, $S_{X}(t)=u S_{Y}(t)$ for all $t>0$. If $g(x)=x^{r}, r>0$, then

$$
\mathrm{H}[X]=u^{r} \mathrm{H}[Y]=\mathrm{H}[I] \mathbf{H}[Y]
$$

Thus, if $g$ is a power function, $g(x)=x^{r}$, then $H$ satisfies Axioms 1-5. 
The "only if" part. First, Axiom 5 implies that $\mathrm{H}[1]=\mathrm{H}[1] \mathrm{H}[1]$ (by letting $I$ and $Y$ be degenerate at 1 ) and, thus, $\mathrm{H}[1]=1$. By Theorem 3, Axioms 1-5 imply that there exists a unique distortion function $g:[0,1] \rightarrow[0,1]$ such that

$$
\mathrm{H}[X]=\int_{0}^{\infty} g\left[S_{X}(t)\right] d t .
$$

We need to show that Axiom 5 implies that $g$ is a power function.

We choose the following claim severity distribution:

$$
\operatorname{Pr}\{Y=1\}=1-w, \quad \operatorname{Pr}\{Y=2\}=w, \quad 0<w<1 .
$$

Note that $Y$ has the decumulative distribution function:

$$
S_{Y}(t)= \begin{cases}1, & \text { if } 0 \leq t<1, \\ w, & \text { if } 1 \leq t<2, \\ 0, & \text { if } 2 \leq t<\infty,\end{cases}
$$

and $X=I Y$ has the decumulative distribution function:

$$
S_{X}(t)= \begin{cases}q, & \text { if } 0 \leq t<1, \\ q w, & \text { if } 1 \leq t<2, \\ 0, & \text { if } 2 \leq t<\infty .\end{cases}
$$

From Theorem 3, the market price for $Y$ is

$$
\mathrm{H}[Y]=\int_{0}^{1} g(1) d t+\int_{1}^{2} g(w) d t=1+g(w),
$$

and the market price for $X=I Y$ is

$$
\mathrm{H}[X]=\int_{0}^{1} g(q) d t+\int_{1}^{2} g(q w) d t=g(q)+g(q w) .
$$

From Axiom 5, we have $\mathrm{H}[X]=\mathrm{H}[I \mathrm{H}[Y]]$, or equivalently

$$
g(q)+g(q w)=g(q)[1+g(w)], \quad 0<q, w<1,
$$

which yields that

$$
g(q w)=g(q) g(w), \quad 0<q, w<1 .
$$

This is a well-known functional equation. From Aczél (1969, p.53), $g$ is a power function, $g(x)=x^{r}$ with $r>0$. 
Wang (1995) proposes a PH-transform principle of premium calculation as follows:

$$
\mathrm{H}[X]=\int_{0}^{\infty} S_{X}(t)^{\frac{1}{2}} d t,
$$

where the exogenous index $\rho \geq 1$ represents the level of aversion toward uncertainty. Note that the PH-transform principle (2) is formula (1) with $r$ restricted to the interval $(0,1]$. Axioms 1-5 provide an axiomatic justification for the PH-transform principle. Wang (1996b) examines some experimental insurance premium data and shows evidence for the power distortion function.

Closely related to the Choquet integral representation

$$
\mathrm{H}[X]=\int_{0}^{\infty} g\left[S_{X}(t)\right] d t
$$

are two economic theories - those of Yaari (1987) and Schmeidler (1989). Both economists use individual risk preference arguments, instead of using market arguments as we do in this paper. The major difference between the Schmeidler model and the Yaari model lies in the interpretation (Quiggin, 1993). Yaari assumes that the objective distributions are known and one applies a distortion to the objective distribution. Schmeidler starts with acts, or risk preferences, and then infers a subjective non-additive set function. This difference in interpretation leads to different methods of conditioning risks upon given information: Following Yaari's theory one updates the objective distribution and then applies a distortion to the conditional predictive distribution. On the other hand, following Schmeidler's theory, one updates the non-additive measure directly. Wang and Young (1996) show that consistency between the two theories in conditioning risks using the Bayes' update rule requires that the distortion function $g$ be a power function.

\section{Analogy with the principle of no arbitrage}

Dybvig and Ross (1992) discuss pricing of risky assets via the principle of no arbitrage no strategy exists that guarantees a positive payoff in some contingency with no chance of a negative payoff and with no net investment. They show that in many cases, the absence of arbitrage is equivalent to the existence of a positive linear pricing rule; this is the first part of the Fundamental Theorem of Asset Pricing. Our axioms lead to a pricing rule 
that is not necessarily additive because we require additivity for comonotonic risks only, Axiom 3. Thus, one may think of our theory as a less restrictive version of the principle of no-arbitrage; we assume no arbitrage for comonotonic risks only.

Hickman and Young (1994) point out the parallel between no arbitrage and the axiom for consistency in subjective probability. The Italian actuary, de Finetti, proposed that one could determine a person's subjective probability as follows: The probability of an event $E, P(E)$, is the amount of money the person is willing to pay in exchange for 1 unit of money if the event $E$ were to occur. One requires that the person is also willing to accept the reverse bet. Consistency is assured by not allowing the person to set up a series of gambles that guarantees a gain for at least one outcome and no loss for any outcome; that is, arbitrage is not permitted. This absence of arbitrage is a key consistency requirement fọ individuals assigning probabilities and is a characteristic of linear pricing rules, by the Fundamental Theorem of Asset Pricing.

There is also a parallel between our market pricing theory and subjective non-additive probability for individual risk assessment, as proposed by Schmeidler (1989) and Yaari (1987). Schmeidler and Yaari relax the independence axiom of EU theory so that it applies only to comonotonic risks. With respect to de Finetti's model, this relaxation of the independence axiom essentially means that consistency, or no arbitrage, is required only for comonotonic risks. In Schmeidler's theory, if the individual's utility function is linear, then the resulting subjective certainty equivalent of a risk is the Choquet integral of the risk with respect to a monotone set function, as is the market insurance price in our Theorem 1 (due to Greco). Yaari's axioms are similar to those of Schmeidler, but he requires that the subjective certainty equivalent of a constant be that constant. Therefore, in Yaari's theory, the subjective certainty equivalent of a risk is the Choquet integral of the risk with respect to a distorted probability, as is the market premium in our Theorem 3 . Thus, we see that there is a parallel between individual risk assessment in the theories of Schmeidler and Yaari and the insurance market pricing rules we develop in this paper, just as there is a parallel between consistency of subjective probabilities in the theory of de Finetti and the principle of no arbitrage in pricing assets.

At the time of writing of this paper, Chateauneuf et al. (1996) consider properties of pricing rules in financial markets with frictions. They obtain a Choquet integral representa- 
tion and show that, among other things, their non-linear pricing rule can explain violation of put-call parity. Artzner et al. (1996) consider desirable properties of a risk measure and maintain that the Choquet integral can be used as a risk measure. We point out that the results of this paper can be applied to pricing in financial markets with frictions as well as to measuring risk.

Acknowledgments: We thank two anonymous referees for helpful suggestions. We also thank Richard D. Phillips and Gordon E. Willmot for comments. The research of the first author was supported by a grant from the Actuarial Education and Research Fund.

\section{References}

Aczél, J. (1969). On Applications and Theory of Functional Equations, Academic Press, New York.

Artzner, P., Delbaen, F., Eber, J.M., and Heath, D. (1996). A characterization of measures of risk. Preprint 1996/14, Department of Mathematics, ULP Strasbourg.

Albrecht, P. (1992). Premium calculation without arbitrage - Discussion of Venter's paper, ASTIN Bulletin, 22, 247-254.

Chateauneuf, A., Kast, R., and Lapied, A. (1996). Choquet pricing for financial markets with frictions, Mathematical Finance 6, 323-330.

Denneberg, D. (1991). Preference reversal and the symmetric Choquet integral, 16th Symposium on Operations Research, University of Trier, 9-11 September, 1991.

Denneberg, D. (1994). Non-Additive Measure And Integral, Kluwer Academic Publishers, Boston.

Dybvig, P.H. and Ross, S.A. (1992). Arbitrage, in The New Palgrave Dictionary of Money and Finance, Vol. 1, 43-50, edited by P.K. Newman, M. Milgate, and J. Eatwell, Macmillan, London. 
Hadar, J. and Russell, W. (1969). Rules for ordering uncertain prospects, American Economic Review 59(1), 25-34.

Hickman, J.C. and Young, V.R. (1994). Discussion of H.U. Gerber and E.S.W. Shiu - "Option pricing by Esscher transforms", Transactions of the Society of Actuaries, Vol. XLVI, 99-140.

Hürlimann, W. (1994). Splitting risk and premium calculation. Bulletin of the Swiss Association of Actuaries, 167-197.

Kaas, R., van Heerwaarden, A.E., and Goovaerts, M.J. (1994). Ordering of Actuarial Risks, Education Series 1, CAIRE, Brussels.

Meyers, G.G. (1991). The competitive market equilibrium risk load formula for increased limits ratemaking, Proceedings of the Casualty Actuarial Society, Vol. LXXVIII, 163200.

Quiggin, J. (1993). Generalized Expected Utility Theory: the rank-dependent model, Kluwer Academic Publishers, Boston.

Schmeidler, D. (1986). Integral representation without additivity. Proceedings of the American Mathematical Society, 97, 255-261.

Schmeidler, D. (1989). Subjective probability and expected utility without additivity, Econometrica, 57, 571-587.

Venter, G.G. (1991). Premium calculation implications of reinsurance without arbitrage, ASTIN Bulletin, 21, 223-230.

von Neumann, J. and Morgenstern, O. (1947). Theory of Games and Economic Behavior, (2nd edition). Princeton, NJ: Princeton University Press.

Wang, S. (1995). Insurance pricing and increased limits ratemaking by proportional hazards transforms, Insurance: Mathematics and Economics, 17, 43-54.

Wang, S. (1996a). Premium calculation by transforming the layer premium density, ASTIN Bulletin, 26, 71-92. 
Wang, S. (1996b). Ambiguity-aversion and the economics of insurance, presented at the Risk Theory Seminar at Wisconsin-Madison in April 1996, submitted to North American Actuarial Journal.

Wang, S. and Young, V.R. (1996). Risk-adjusted credibility premiums using distorted probabilities, submitted to Scandinavian Actuarial Journal.

Yaari, M.E. (1987). The dual theory of choice under risk, Econometrica, 55, 95-115.

\section{Appendix A: Extension to real-valued random variables}

Let the set of insurance risks, $\mathcal{X}$, be the collection of real-valued random variables on the measure space $(\Omega, \mathcal{A}, P)$. Extend the continuity axiom, Axiom 4, to include the third condition $\lim _{d \rightarrow-\infty} \mathrm{H}[\max (X, d)]=\mathrm{H}[X]$.

If we assume that $\mathrm{H}$ satisfies Axioms 2 through 4 (extended), then we can represent $\mathrm{H}$ via the Choquet integral with respect to the monotone set function given in Theorem 1. Indeed, let $X \in \mathcal{X}$ and $M<0$; then, $\max (X, M)-M$ is a non-negative random variable. Because $-M$, a constant, is comonotonic with every risk, and because monotonicity and comonotonic additivity imply positive homogeneity of $\mathbf{H}$, we have that

$$
\mathrm{H}[\max (X, M)-M]=\mathrm{H}[\max (X, M)]+\mathrm{H}[-M]=\mathrm{H}[\max (X, M)]-M \mathrm{H}[1] .
$$

Thus, $\mathrm{H}[\max (X, M)]=\mathrm{H}[\max (X, M)-M]+M \mathrm{H}[1]$.

By Greco's Representation Theorem applied to $\max (X, M)-M$, we have that

$$
\begin{aligned}
\mathrm{H}[\max (X, M)] & =\int[\max (X, M)-M] d \gamma+M \gamma(\Omega) \\
& =\int[\max (X, M)] d \gamma-M \gamma(\Omega)+M \gamma(\Omega) \\
& =\int[\max (X, M)] d \gamma .
\end{aligned}
$$

Now,

$$
\begin{aligned}
\mathrm{H}[X] & =\lim _{M \rightarrow-\infty} \mathrm{H}[\max (X, M)] \\
& =\lim _{M \rightarrow-\infty}\left[\int_{-\infty}^{0}\{\gamma(\max (X, M)>t)-\gamma(\Omega)\} d t+\int_{0}^{\infty} \gamma(\max (X, M)>t) d t\right] \\
& =\lim _{M \rightarrow-\infty}\left[\int_{-\infty}^{M}\{\gamma(\Omega)-\gamma(\Omega)\} d t+\int_{M}^{0}\{\gamma(X>t)-\gamma(\Omega)\} d t+\int_{0}^{\infty} \gamma(X>t) d t\right] \\
& =\lim _{M \rightarrow-\infty} \int_{M}^{0}\{\gamma(X>t)-\gamma(\Omega)\} d t+\int_{0}^{\infty} \gamma(X>t) d t \\
& =\int_{-\infty}^{0}\{\gamma(X>t)-\gamma(\Omega)\} d t+\int_{0}^{\infty} \gamma(X>t) d t \\
& =\int X d \gamma .
\end{aligned}
$$


in which the next-to-the-last equality follows from continuity of the Choquet integral, (Denneberg, 1994, Proposition 1.6). Thus, we can represent $H$ as the Choquet integral with respect to a monotone set function.

Note that Theorem 2 can be extended in a similar fashion to real-valued random variables by replacing $\mathrm{H}[1](g \circ P)$ for $\gamma$ in the above. With the condition $\mathrm{H}[1]=1$, the Choquet integral of a real-valued random $X$ with respect to a distortion $g$ is

$$
\mathrm{H}[X]=\int X d(g \circ P)=\int_{-\infty}^{0}\left\{g\left[S_{X}(t)\right]-1\right\} d t+\int_{0}^{\infty} g\left[S_{X}(t)\right] d t,
$$

which is asymmetric in that $\mathrm{H}[-X] \neq-\mathrm{H}[X]$. Denneberg (1991) examines a pricing functional similar to the one above, except that he requires it to be symmetric: $H[-X]=$ $-\mathbf{H}[X]$. 
